\title{
Active surveillance of women diagnosed with atypical ductal hyperplasia on core needle biopsy may spare many women potentially unnecessary surgery, but at the risk of undertreatment for a minority: 10-year surgical outcomes of 114 consecutive cases from a single center
}

\author{
Gelareh Farshid ${ }^{1}$, Suzanne Edwards ${ }^{2}$, James Kollias ${ }^{3}$ and Peter Grantley Gill ${ }^{3}$ \\ ${ }^{1}$ Surgical Pathology, BreastScreen SA, Discipline of Medicine, Adelaide University and South Australian \\ Pathology, Frome Road Adelaide University and Directorate of Surgical Pathology, Adelaide, SA, Australia; \\ ${ }^{2}$ Adelaide Health Technology Assessment, School of Public Health, Adelaide University, Adelaide, SA, \\ Australia and ${ }^{3}$ BreastScreen $S A$ and The Department of Surgery, University of Adelaide, Adelaide, SA, \\ Australia
}

A needle core biopsy diagnosis of atypical ductal hyperplasia is an indication for open biopsy. The launch of randomized clinical trials of active surveillance for low-risk ductal carcinoma in situ leads to the paradoxical situation of women with low-grade ductal carcinoma in situ being observed, whereas those with atypical ductal hyperplasia have surgery. If the malignancies diagnosed after surgery for atypical ductal hyperplasia are dominated by low-risk ductal carcinoma in situ, women with atypical ductal hyperplasia may also be considered for surveillance. This 10-year prospective observational study includes women diagnosed with atypical ductal hyperplasia on core biopsy after screening mammography. We retrieved their clinical, imaging and histologic data and carried out a blind review of core biopsy histology, sub-classifying the atypical ductal hyperplasia along a spectrum from hyperplasia to ductal carcinoma in situ. Using the final surgical pathology data, we calculated: (1) The proportion and grades of ductal carcinoma in situ and invasive cancers diagnosed at open biopsy. (2) The histologic extent of the malignancy at surgery. (3) The biomarker profile and nodal status of any invasive cancers. (4) Ascertained any independent predictors of (i) any malignancy, (ii) high-risk malignancy, defined in this study as invasive cancer, or high-grade ductal carcinoma in situ, or intermediate grade ductal carcinoma in situ with any necrosis. (5) Extrapolated the above to simulate active surveillance for women with screen-detected atypical ductal hyperplasia. Between January 2005 and December 2014, 114 women, mean age 59 years (range 40-79 years) were included. Surgical pathology, available in 110 (97\%), confirmed malignancy in $46(40 \%)$. All 46 malignant cases had ductal carcinoma in situ, accompanied by invasive carcinoma in 9 (8\%) women. Together, 21 $(19 \%)$ women had either invasive cancer $(9 \%)$, high-grade ductal carcinoma in situ $(6 \%)$, or necrotizing, intermediate grade ductal carcinoma in situ (6\%). Only one of nine invasive breast cancers was grade 1, 3 were multifocal, all were $\leq \mathbf{8 m m}$, node negative, and ER positive but two were HER2 amplified. The mean extent of the ductal carcinoma in situ in any one specimen was $19.8 \mathrm{~mm}$, median $13 \mathrm{~mm}$, range $2-110 \mathrm{~mm}$. Overall 32 women, $29 \%$ of the whole cohort and $70 \%$ of those $\mathbf{4 6}$ with malignancy, required further surgery, including mastectomy in $12(11 \%)$. A multivariable model for predicting the likelihood of any malignancy showed a statistically significant association only with the post review subtype of atypical ductal hyperplasia, adjusting for lesion size. Independent predictors of high-risk malignancy (invasive cancer or non-low-grade ductal carcinoma in situ) were not identified. If active surveillance is adopted for screen-detected atypical ductal hyperplasia diagnosed on core

Correspondence: Professor G Farshid, MBBS, MPH, MD, FRCPA, Surgical Pathology, BreastScreen SA, Discipline of Medicine, Adelaide University and South Australian Pathology, Frome Road Adelaide University and Directorate of Surgical Pathology, Adelaide, SA 5000, Australia.

E-mail: gelareh.farshid2@sa.gov.au

Received 19 May 2017; revised 23 July 2017; accepted 23 July 2017; published online 3 November 2017 


\begin{abstract}
biopsy, $60 \%$ of women will avoid unnecessary surgery and a further $24 \%$ would meet eligibility criteria for ductal carcinoma in situ surveillance trials. However, $18 \%$ of women will have undiagnosed invasive breast cancer or nonlow-risk ductal carcinoma in situ. These women with high-risk lesions are not reliably identified pre-operatively. Modern Pathology (2018) 31, 395-405; doi:10.1038/modpathol.2017.114; published online 3 November 2017
\end{abstract}

Atypical intraductal epithelial proliferations diagnosed on needle core biopsy are often referred to as atypical ductal hyperplasia in the literature. ${ }^{1}$ In this context, the term atypical ductal hyperplasia covers a broad spectrum of lesions that, while showing some atypical histologic features, fall short of the diagnostic criteria for ductal carcinoma in situ. Prompt surgical biopsy is usually recommended for this group of lesions, owing to their high upgrade rates to ductal carcinoma in situ or invasive cancer. Even in contemporary practice, reflected in studies published since 2011 (Table 1), the upgrade rates for atypical ductal hyperplasia diagnosed on core biopsy range from $9-56 \% .^{2-14}$

To our knowledge, at present four randomized clinical trials of active surveillance for so called 'low-risk ductal carcinoma in situ' are at various stages of activation. These include the UK LORIS trial,15 LORD (ClinicalTrials.gov Identifier: NCT02492607), ${ }^{16}$ COMET (ClinicalTrials.gov Identifier: NCT02926911), and the Australian LARRAKIN trial. With LORIS as the exemplar, these trials seek to determine whether active surveillance by mammography may be a non-inferior alternative to surgical excision for women diagnosed with low grade or intermediate grade, non-necrotic ductal carcinoma in situ, diagnosed on core biopsy or diagnostic open biopsy without clear margins. Eligibility requirements for LORIS include age $\geq 46$ years, detection through screening or incidental (asymptomatic) microcalcifications and absence of (i) any symptoms, (ii) prior history of breast cancer, or (iii) inclusion in groups at high risk for breast cancer. Women randomized to the active surveillance arm of the LORIS trial are monitored by annual mammography. Surgery will be recommended if imaging features suspicious for invasion develop or if the

Table 1 Reported upgrade rates for atypical ductal hyperplasia diagnosed on needle core biopsy since 2011

\begin{tabular}{lrc} 
Author, year & $\mathrm{N}$ & Upgrade \\
\hline Khoury $^{9}$ & 203 & $28.30 \%$ \\
Mesurolle $^{12}$ & 50 & $56 \%$ \\
Gumus $^{6}$ & 105 & $41.7 \%$ (NCB), $20.6 \%$ (VAB) \\
Polom $^{14}$ & 134 & $9 \%$ \\
McGhan $^{11}$ & 114 & $18 \%$ \\
Kim $^{10}$ & 85 & $37 \%$ \\
Hsu $^{8}$ & 134 & $39.6 \%$ \\
Bendifallah $^{4}$ & 107 & $19 \%$ \\
Nguyen $^{13}$ & 140 & $11.4 \%$ \\
Hong $^{7}$ & 124 & $45.2 \%$ \\
Deshaies $^{5}$ & 422 & $31.3 \%$ \\
Ancona & & $19.4 \%$ \\
Allison $^{2}$ & 98 & $20.6 \%$ \\
\hline
\end{tabular}

microcalcifications extend beyond the original quadrant. The primary outcome measure in this trial is the ipsilateral invasive breast cancer-free survival rate at 5 years. Annual mammography will continue for at least 10 years and a range of secondary outcome measures will also be evaluated, including time to the development of ipsilateral breast cancer, to any surgery and to mastectomy. A range of patient reported outcomes, notably quality of life measures and health economic analyses, comprise important facets of this trial.

The launch of surveillance trials for ductal carcinoma in situ leads to the paradoxical situation whereby women with atypical ductal hyperplasia would be advised to proceed promptly to surgery while those with established ductal carcinoma in situ are being observed. In this context, our aim was to determine the frequency and nature of malignancies diagnosed after surgery for screen-detected atypical ductal hyperplasia on core biopsy. If the surgical outcomes in this group are dominated by low-risk ductal carcinoma in situ, atypical ductal hyperplasia may be included in trials of active surveillance, noting that low-grade ductal carcinoma in situ represents $<15 \%$ of all screen-detected ductal carcinoma in situ. ${ }^{17,18}$

Aims: we wished to evaluate our population-based screening service's experience with lesions diagnosed as atypical ductal hyperplasia on core biopsy after assessment of screen-detected breast lesions. Specifically, we were interested in the following outcome measures:

(1) The proportion and grades of ductal carcinoma in situ and invasive cancers diagnosed at open biopsy.

(2) The histologic extent of the malignancies at surgery.

(3) The biomarker profile and nodal status of any invasive cancers.

(4) Ascertainment of independent predictors of (i) any malignancy, (ii) high-risk malignancy, defined in this study as invasive cancer, or high-grade ductal carcinoma in situ, or intermediate grade ductal carcinoma in situ with any necrosis.

(5) Extrapolation of the above to simulate a policy of active surveillance for women with screendetected atypical ductal hyperplasia.

\section{Materials and methods}

\section{The Design of our Breast Cancer Screening Program}

The setting for this audit is BreastScreen South Australia. BreastScreen South Australia is part of a national breast cancer screening program, aimed at 
asymptomatic women 50-69. Recently, the age range has been extended to women aged 74. As described previously, ${ }^{19}$ two yearly screening mammograms and mandatory double reading are implemented, using the Tabar imaging grading scheme. ${ }^{20}$ Women with lesions graded 3 and above are recalled for further assessment. After further imaging work-up, the lesion is re-graded and biopsy is performed for lesions with post work-up grades of 3 and above. For non-calcified lesions, we use ultrasound guided core biopsies and usually retrieve three cores of tissue. Vacuum-assisted core biopsy is used for microcalcifications. We use immediate digital specimen X-ray to ensure calcifications are retrieved. Open biopsy is recommended when the needle biopsy findings are indefinite or discordant with the imaging, or the core biopsy finds a borderline lesion. For lesions undergoing surgery, all final pathology is captured prospectively into a database. Clients not referred for surgery are tracked through their subsequent screening visits and via the State Cancer Registry, which notifies us of breast cancers diagnosed outside the Service in screened women. ${ }^{21}$

We routinely obtain client consent prospectively for the use of de-identified data for service monitoring and quality improvement purposes. Our institutional review board does not require additional consent for clinical audits.

\section{Study Design}

For a 10-year consecutive time period from Jan 2005 to Dec 2014 we included women with a diagnosis of atypical ductal hyperplasia as the most advanced lesion on core biopsy. Demographic and clinical data were collected prospectively. These include client age and round (episode number) of screening. Among imaging variables, we retrieved the dominant imaging morphology, lesion extent, Tabar imaging grade, the biopsy modality (ultrasound or stereotactic) and the needle core biopsy method (conventional $14 \mathrm{G}$ core biopsy or $11 \mathrm{G}$ vacuum-assisted core biopsy). We included the original core biopsy diagnosis. Unaware of the final surgical outcomes, we also performed a retrospective histologic review of the core biopsies, whereby one of us (GF), determined the number of cores submitted, the extent of atypia on the core biopsy and classified the atypia into a five-tier system, consisting of atypia bordering florid hyperplasia, complex architecture dominated by micropapillary tufting (as opposed to the classic cribriform architecture of atypical ductal hyperplasia), conventional atypical ductal hyperplasia, architectural atypia bordering ductal carcinoma in situ and significant cytologic atypia.

Through chart review, we documented the findings on breast surgery. This included the final surgical pathologic diagnosis, categorized as invasive cancer, ductal carcinoma in situ, atypical ductal hyperplasia or non-malignant epithelial proliferations. For women with malignancy, the extent of the malignancy, the need for subsequent surgeries and the outcomes of those procedures were documented.

All findings were entered into a database for statistical analysis. Using the JMP 12 and the SAS 9.4 statistical software packages, we performed descriptive analyses of the data. In addition, we ascertained independent predictors of (i) any malignancy, (ii) high-risk malignancy, defined in this study as invasive cancer, or high-grade ductal carcinoma in situ, or intermediate grade ductal carcinoma in situ with any necrosis.

To find these independent predictors, we initially performed 11 univariate logistic regression models to test if any of these covariates were associated with the likelihood of malignancy. To test if the covariates were associated with the likelihood of high-risk malignancy, 11 further univariate logistic regression models were performed. An initial multivariable model was created for the outcome likelihood of malignancy versus all covariates with $P$-value $<0.2$ on univariate regression. Backward elimination was performed, deleting the variable with the highest $P$ value, one model at a time until all covariates had $P$ values $<0.1$. This procedure was repeated for the outcome likelihood of high-risk malignancy.

\section{Results}

Between January 2005 and December 2014, 114 women diagnosed with screen-detected atypical ductal hyperplasia on core biopsy are included. The mean patient age was 59 years (range 40-79 years). In 29 women (26\%) the atypical ductal hyperplasia was diagnosed at the first screening episode, in 84 women (74\%) in the 2nd to 16th screening visits and this information was missing in one case.

Microcalcifications comprised the dominant screen-detected lesion in 95 (83\%) women, one case showed a mass with calcifications, while noncalcified lesions, such as a discrete or stellate mass, architectural distortion or asymmetric density were the main imaging finding in 18 (16\%) cases.

Overall, the imaging grade was classified as 3 (indeterminate) in $76(67 \%)$ cases, 4 (suspicious) in $24(21 \%)$ and 5 (highly suspicious) in 14 (12\%). Among microcalcifications, the breakdown of the imaging grade was $72 \%$ grade $3,19.0 \%$ grade 4 and $10 \%$ grade 5 .

The mean imaging lesion extent was $19.3 \mathrm{~mm}$, median $11 \mathrm{~mm}$, range of $3-150 \mathrm{~mm}$. Vacuumassisted core biopsy, 11G, was performed in 95 $(83 \%)$ cases and conventional $14 \mathrm{G}$ ultrasound guided core biopsy in $19(17 \%)$ cases. The number of cores retrieved was $<10$ in 25 cases, $\geq 10$ in 83 cases and not available in six cases. The number of foci involved by $\mathrm{ADH}$ was $\leq 3$ in 54 cases (51\%) and $>3$ in 53 cases $(49 \%)$. 
After a core biopsy diagnosis of atypical ductal hyperplasia, diagnostic open biopsy was recommended by the multidisciplinary team for all women. Surgical outcomes are available in 110 cases $(97 \%)$. No surgery was performed in three cases, whereas the surgical specimen did not reach the laboratory in one case. The reason for not proceeding with surgery were severe comorbidities in one case,
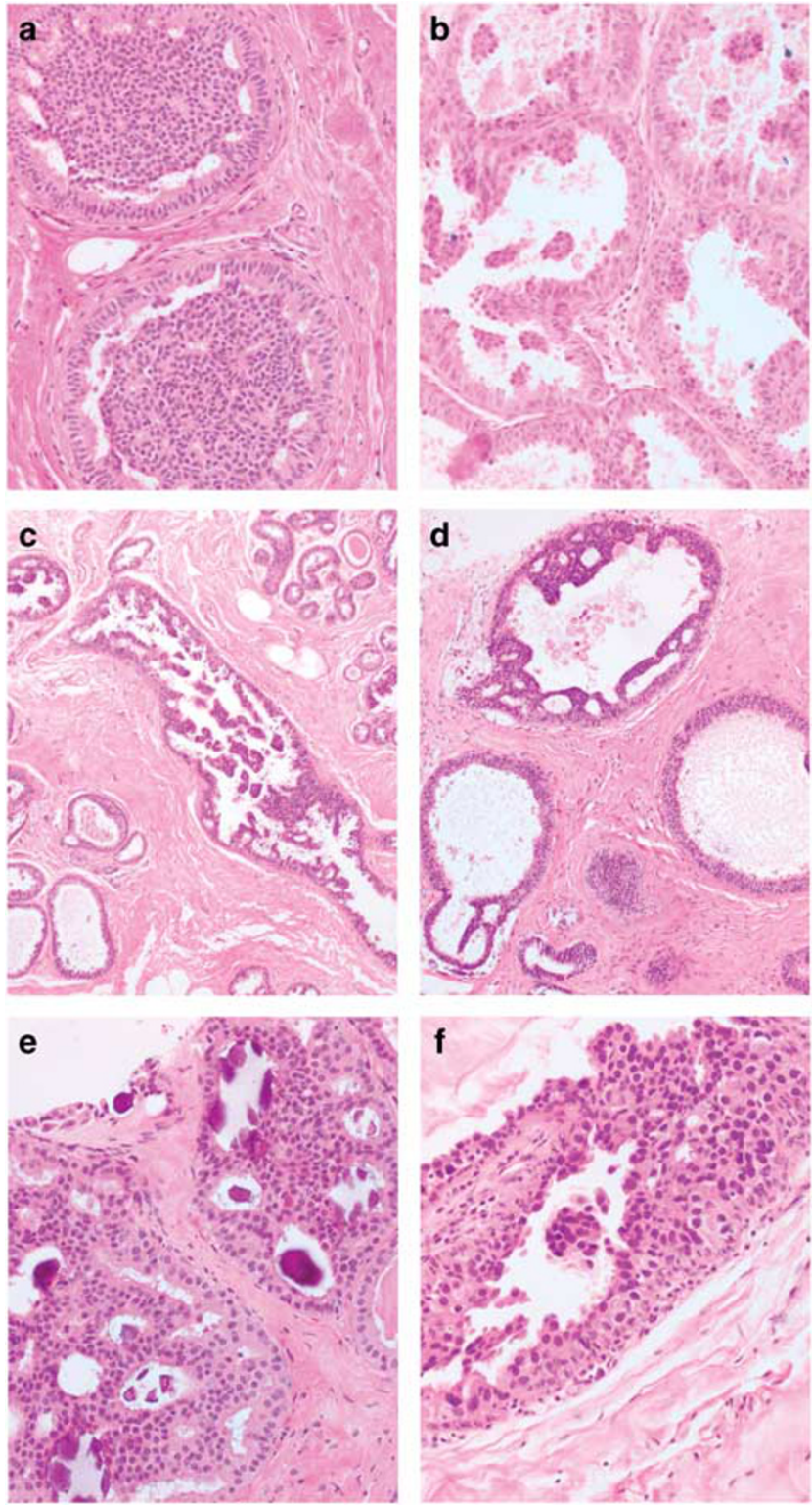

Figure $1(\mathbf{a}-\mathbf{f})$ The range of atypical intraductal proliferative lesions on histology. On review a range of histologic lesions was classified as atypical. These included (a) cases bordering on usual hyperplasia, (b) a subset dominated by a micropapillary architecture, $(\mathbf{c}, \mathbf{d})$ the majority of cases full filling the diagnostic criteria for classic atypical ductal hyperplasia as defined from surgical specimens, (e) more established, extensive lesions, bordering on low-grade ductal carcinoma in situ, and (f) lesions with greater cytologic atypia than is usually seen in atypical ductal hyperplasia. removal of all microcalcifications by core biopsy in another case and unknown in the final case. Mammographic surveillance at 50 months in two women and 12 months in the third woman has shown no progression. One woman's surgical specimen underwent specimen imaging, which documented the removal of microcalcifications but thereafter the specimen did not reach the laboratory. No follow-up is available on this case. Overall, surgical or imaging follow-up is therefore available in 113 of 114 cases $(99 \%)$.

Histologic review, carried out unaware of the surgical biopsy findings, confirmed that cases with the cyto-architectural features of classic atypical ductal hyperplasia comprised the largest subset, composing $47(46 \%)$ of the cohort. In 24 cases, the features bordered hyperplasia, five had a mostly micropapillary architecture, 16 had architectural complexity and extent that bordered ductal carcinoma in situ, and nine exhibited cytologic atypia exceeding that associated with low-grade ductal carcinoma in situ (Figure 1).

\section{Surgical Pathology Findings}

Diagnostic open biopsy was performed in 111 of 114 women (97\%). Malignancy was identified in 46 cases $(40 \%)$, further atypia was the most advanced lesion in 40 cases $(36 \%)$, and benign, non-atypical findings were reported in the remaining 28 cases (25\%). Results are unavailable in one case due specimen misplacement. The upgrade rates for the review tiers of atypia is shown in Table 2. As can be seen there is a stepwise progression in the risk of an upgrade.

Among the 46 malignant cases, ductal carcinoma in situ was identified in all cases. This was accompanied by invasive carcinoma in nine cases $(20 \%)$. In five of the nine invasive cancers, microcalcifications comprised the main imaging finding, whereas lesions other than calcifications were found in the remaining four cases. Among the invasive cancers, the imaging grade was grade 3 (indeterminate) in four cases, grade 4 (suspicious) in four cases and grade 5 in the last case. The mean patient age was 60 years. The mean lesion extent on imaging was $15.8 \mathrm{~mm}$, range $5-35 \mathrm{~mm}$. The ductal carcinoma in situ component of the invasive cancers was low grade in two cases, intermediate nuclear

Table 2 Upgrade rates by review histologic subtype of atypical ductal hyperplasia

\begin{tabular}{lrc}
\hline Review subgroup & $\mathrm{N}$ & $\%$ Malignant \\
\hline Bordering hyperplasia & 24 & $20.8 \%$ \\
Micropapillary & 5 & $0.0 \%$ \\
ADH & 47 & $40.4 \%$ \\
ADH-bordering DCIS & 16 & $68.8 \%$ \\
Cytologic atypia & 9 & $66.7 \%$ \\
\hline
\end{tabular}


grade without necrosis in four cases and high-grade ductal carcinoma in situ in three cases. The extent of ductal carcinoma in situ was $<15 \mathrm{~mm}$ in five cases $(4,4,7,7,7,8 \mathrm{~mm})$ and larger than this in four cases $(20,30,40,45 \mathrm{~mm})$. The invasive carcinoma was unifocal in six, and multifocal in three cancers. The multifocal cancers comprised one invasive lobular carcinoma, one case with two foci of invasive mucinous carcinoma and one woman with multiple foci of invasive ductal carcinoma, together with a tubular carcinoma and a focus of invasive lobular carcinoma. The invasive cancers were all smaller than $10 \mathrm{~mm}$, specifically the largest focus measured $2,2,3,4,4,5,7,7,8$, and $8 \mathrm{~mm}$. Two invasive cancers were grade 1 , six were grade 2 , and two were grade 3 cancers. All were node negative and ER positive, while 2 also showed HER2 amplification by in situ hybridization.

Inclusive of the cases with areas of invasion, the ductal carcinoma in situ was low grade in 17 cases $(37 \%)$, intermediate nuclear grade without necrosis in 15 cases (33\%), intermediate nuclear grade with foci of necrosis in five cases $(12 \%)$ and high-grade in nine cases $(20 \%)$. Together low grade and intermediate nuclear grade ductal carcinoma in situ without necrosis comprised 32 of the 46 cases (70\%).

Among the 37 cases with pure ductal carcinoma in situ, the lesion grade was low grade in 15 (41\%), intermediate grade without necrosis in $11(30 \%)$, intermediate grade with necrosis in $5(14 \%)$ and high-grade in 6 (16\%). Overall, 21 women, comprising $19 \%$ of the study population had either invasive breast cancer, ${ }^{9}$ high-grade ductal carcinoma in situ, ${ }^{6}$ or necrotizing intermediate grade ductal carcinoma in situ. ${ }^{6}$

Considering all malignant cases, the mean extent of the ductal carcinoma in situ in any one specimen was $19.8 \mathrm{~mm}$, median $13 \mathrm{~mm}$, range $2-110 \mathrm{~mm}$. However, this is likely an underestimation as many women had several surgical procedures due to positive or close margins in the initial open biopsy.

Overall 32 of the 46 women $(70 \%)$ with malignancy, representing $29 \%$ of the whole cohort, required further breast surgery. The additional surgery took the form of a breast re-excision in 19 cases and mastectomy in 12 cases (26\%), including a case of bilateral mastectomy for an incidental, contralateral breast cancer. Only 15 women (33\%) did not require breast surgery beyond the initial open biopsy, but one of these women did proceed with sentinel node biopsy.

Axillary staging was undertaken in 18 women, including all 9 with concurrent invasive breast cancer. The node count was 4 or fewer in 14 of these 18 cases. All nodes were negative.

$<$ !?A3B2 tlsb $-1 \%$ ? $>$ The flow chart in Figure 2 shows the outcomes, if the inclusion criteria for lowrisk ductal carcinoma in situ trials were applied to this cohort of women with atypical ductal hyperplasia diagnosed on core biopsy. Of the 110 women with surgical outcome data, 28 had benign findings,

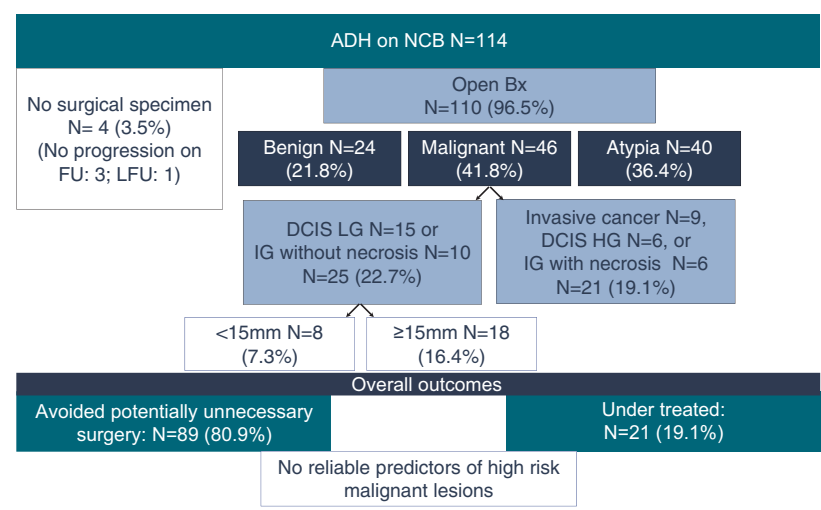

Figure 2 Flow chart of findings after follow-up of 114 cases of screen-detected atypical ductal hyperplasia diagnosed on needle core biopsy to simulate a policy of active surveillance.

40 only further atypia, 15 low-grade ductal carcinoma in situ and 10 intermediate grade ductal carcinoma in situ without necrosis. Taken together, this policy would avoid potentially unnecessary surgery for these 89 women, representing $81 \%$ of the cohort. However, this policy would lead to under-treatment of the remaining 21 women (19\%), comprising 9 women with invasive cancer, 6 with high-grade ductal carcinoma in situ and 6 with intermediate grade ductal carcinoma in situ with necrosis.

\section{Statistical Analysis}

As shown in Table 3, statistically significant association were found between the outcome likelihood of malignancy and review histologic subgroup $(P=0.0349)$, number of cores retrieved (global $P$ value $=0.0217$ ) and increasing lesion size on imaging (global $P$-value $=0.0405$ ). Regarding the number of cores retrieved, for every increase of one core biopsy, the odds of malignancy increased by $18 \%$ (Odds Ratio $=1.18$, 95\% CI: 1.03, 1.37), possibly reflecting lesion size. Quantifying the effect of increasing lesion size, for every unit increase, the odds of malignancy increased by $2.3 \%$ (Odds ratio $=1.023$, 95\% CI: 1.001, 1.0457).

An initial multivariable model was fitted for the outcome likelihood of malignancy versus all covariates with $P$-value $<0.2$ on univariate regression: namely review diagnostic category, number of cores retrieved, screening episode, and size of lesion on mammography. Backwards elimination was performed, deleting variable with the highest $P$-value, one model at a time, until all covariates had $P$-value $<0.1$. Our multivariable models showed a statistically significant association only between likelihood of malignancy and review diagnostic category, adjusting for size of lesion (global $P$-value $=0.0470$ ). The association between likelihood of malignancy and lesion size, adjusting for diagnostic category was almost significant (global $P$-value $=0.0565$ ). 
Table 3 Statistical modeling

\begin{tabular}{|c|c|c|c|c|c|c|c|c|c|}
\hline $\begin{array}{l}\text { Model } \\
\text { number }\end{array}$ & Outcome & Predictor & Comparison & Reference & $\begin{array}{l}\text { Odds } \\
\text { ratio }\end{array}$ & $\begin{array}{c}\text { Lower } 95 \% \\
C L\end{array}$ & $\begin{array}{l}\text { Upper 95\% } \\
\text { CL }\end{array}$ & Comparison P-value & $\begin{array}{l}\text { Global } \\
\text { P-value }\end{array}$ \\
\hline 1 & Any malignancy & $\begin{array}{l}\text { Review ADH } \\
\text { subtype }\end{array}$ & $\mathrm{ADH}$ & Micropapillary & - & - & - & 1.00 & 0.03 \\
\hline 1 & Any malignancy & $\begin{array}{l}\text { Review ADH } \\
\text { subtype }\end{array}$ & $\mathrm{ADH}$ & Bordering DCIS & 0.31 & 0.09 & 1.03 & 0.06 & 0.03 \\
\hline 1 & Any malignancy & $\begin{array}{l}\text { Review ADH } \\
\text { subtype }\end{array}$ & $\mathrm{ADH}$ & Bordering hyperplasia & 2.58 & 0.82 & 8.10 & 0.10 & 0.03 \\
\hline 1 & Any malignancy & $\begin{array}{l}\text { Review ADH } \\
\text { subtype }\end{array}$ & $\mathrm{ADH}$ & Cytologic atypia & 0.34 & 0.08 & 1.53 & 0.16 & 0.03 \\
\hline 1 & Any malignancy & $\begin{array}{l}\text { Review ADH } \\
\text { subtype }\end{array}$ & Micropapillary & Bordering DCIS & - & - & - & 1.00 & 0.03 \\
\hline 1 & Any malignancy & $\begin{array}{l}\text { Review ADH } \\
\text { subtype }\end{array}$ & Micropapillary & Bordering hyperplasia & - & - & - & 1.00 & 0.03 \\
\hline 1 & Any malignancy & $\begin{array}{l}\text { Review ADH } \\
\text { subtype }\end{array}$ & Micropapillary & Cytologic atypia & - & - & - & 1.00 & 0.03 \\
\hline 1 & Any malignancy & $\begin{array}{l}\text { Review ADH } \\
\text { subtype }\end{array}$ & Bordering DCIS & Bordering hyperplasia & 8.36 & 1.97 & 35.46 & 0.00 & 0.03 \\
\hline 1 & Any malignancy & $\begin{array}{l}\text { Review ADH } \\
\text { subtype }\end{array}$ & Bordering DCIS & Cytologic atypia & 1.1 & 0.19 & 6.29 & 0.91 & 0.03 \\
\hline 1 & Any malignancy & $\begin{array}{l}\text { Review ADH } \\
\text { subtype }\end{array}$ & Bordering hyperplasia & Cytologic atypia & 0.13 & 0.02 & 0.72 & 0.02 & 0.03 \\
\hline 2 & Any malignancy & Extent of ADH & $\leq 3$ foci & $>3$ foci & 0.95 & 0.44 & 2.09 & & 0.91 \\
\hline 3 & Any malignancy & Calcs versus mass & Calcs & Mass & 1.29 & 0.46 & 3.57 & & 0.63 \\
\hline 4 & Any malignancy & Imaging grade & Grade 3 & Grade 4 & 0.40 & 0.16 & 1.04 & 0.06 & 0.17 \\
\hline 4 & Any malignancy & Imaging grade & Grade 3 & Grade 5 & 0.75 & 0.24 & 2.41 & 0.63 & 0.17 \\
\hline 4 & Any malignancy & Imaging grade & Grade 4 & Grade 5 & 1.87 & 0.49 & 7.08 & 0.36 & 0.17 \\
\hline 5 & Any malignancy & Core biopsy method & Ultrasound & VAB & 0.59 & 0.21 & 1.69 & & 0.32 \\
\hline 6 & Any malignancy & Number of cores & & & 1.18 & 1.03 & 1.37 & & 0.02 \\
\hline 7 & Any malignancy & $\begin{array}{l}\text { Foci of ADH: } \\
\text { continuous }\end{array}$ & & & 0.99 & 0.89 & 1.10 & & 0.91 \\
\hline 8 & Any malignancy & Year of diagnosis & & & 1.06 & 0.92 & 1.21 & & 0.43 \\
\hline 9 & Any malignancy & Patient age & & & 1.03 & 0.98 & 1.07 & & 0.25 \\
\hline 10 & Any malignancy & Episode of screening & & & 1.08 & 0.96 & 1.20 & & 0.19 \\
\hline 11 & Any malignancy & Imaging lesion size & & & 1.02 & 1.00 & 1.05 & & 0.04 \\
\hline 12 & $\begin{array}{l}\text { High-risk } \\
\text { malignancy }\end{array}$ & $\begin{array}{l}\text { Review ADH } \\
\text { subtype }\end{array}$ & $\mathrm{ADH}$ & Micropapillary & - & - & - & 1.00 & 0.31 \\
\hline 12 & $\begin{array}{l}\text { High-risk } \\
\text { malignancy }\end{array}$ & $\begin{array}{l}\text { Review ADH } \\
\text { subtype }\end{array}$ & $\mathrm{ADH}$ & Bordering DCIS & 0.39 & 0.10 & 1.45 & 0.16 & 0.31 \\
\hline 12 & $\begin{array}{l}\text { High-risk } \\
\text { malignancy }\end{array}$ & $\begin{array}{l}\text { Review ADH } \\
\text { subtype }\end{array}$ & $\mathrm{ADH}$ & Bordering hyperplasia & 1.93 & 0.37 & 10.08 & 0.44 & 0.31 \\
\hline 12 & $\begin{array}{l}\text { High-risk } \\
\text { malignancy }\end{array}$ & $\begin{array}{l}\text { Review ADH } \\
\text { subtype }\end{array}$ & $\mathrm{ADH}$ & Cytologic atypia & 0.35 & 0.07 & 1.74 & 0.20 & 0.31 \\
\hline 12 & $\begin{array}{l}\text { High-risk } \\
\text { malignancy }\end{array}$ & $\begin{array}{l}\text { Review ADH } \\
\text { subtype }\end{array}$ & Micropapillary & Bordering DCIS & - & - & - & 1.00 & 0.31 \\
\hline 12 & $\begin{array}{l}\text { High-risk } \\
\text { malignancy }\end{array}$ & $\begin{array}{l}\text { Review ADH } \\
\text { subtype }\end{array}$ & Micropapillary & Bordering hyperplasia & - & - & - & 1.00 & 0.31 \\
\hline 12 & $\begin{array}{l}\text { High-risk } \\
\text { malignancy }\end{array}$ & $\begin{array}{l}\text { Review ADH } \\
\text { subtype }\end{array}$ & Micropapillary & Cytologic atypia & - & - & - & 1.00 & 0.31 \\
\hline 12 & $\begin{array}{l}\text { High-risk } \\
\text { malignancy }\end{array}$ & $\begin{array}{l}\text { Review ADH } \\
\text { subtype }\end{array}$ & Bordering DCIS & Bordering hyperplasia & 5 & 0.83 & 30.02 & 0.08 & 0.31 \\
\hline
\end{tabular}




\section{Table 3 (Continued)}

\begin{tabular}{|c|c|c|c|c|c|c|c|c|c|}
\hline $\begin{array}{l}\text { Model } \\
\text { number }\end{array}$ & Outcome & Predictor & Comparison & Reference & $\begin{array}{l}\text { Odds } \\
\text { ratio }\end{array}$ & $\begin{array}{c}\text { Lower 95\% } \\
\text { CL }\end{array}$ & $\begin{array}{c}\text { Upper } 95 \% \\
\text { CL }\end{array}$ & Comparison P-value & $\begin{array}{l}\text { Global } \\
\text { P-value }\end{array}$ \\
\hline 12 & $\begin{array}{l}\text { High-risk } \\
\text { malignancy }\end{array}$ & $\begin{array}{l}\text { Review ADH } \\
\text { subtype }\end{array}$ & Bordering DCIS & Cytologic atypia & 0.91 & 0.16 & 5.20 & 0.91 & 0.31 \\
\hline 12 & $\begin{array}{l}\text { High-risk } \\
\text { malignancy }\end{array}$ & $\begin{array}{l}\text { Review ADH } \\
\text { subtype }\end{array}$ & Bordering hyperplasia & Cytologic atypia & 0.18 & 0.02 & 1.35 & 0.10 & 0.31 \\
\hline 13 & $\begin{array}{l}\text { High-risk } \\
\text { malignancy }\end{array}$ & Extent of $\mathrm{ADH}$ & $\leq 3$ foci & $>3$ foci & 1.17 & 0.43 & 3.16 & & 0.76 \\
\hline 14 & $\begin{array}{l}\text { High-risk } \\
\text { malignancy }\end{array}$ & Calcs versus mass & Calcs & Mass & 0.86 & 0.25 & 2.93 & & 0.81 \\
\hline 15 & $\begin{array}{l}\text { High-risk } \\
\text { malignancy }\end{array}$ & Imaging grade & Grade 3 & Grade 4 & 0.6 & 0.20 & 1.83 & 0.37 & 0.65 \\
\hline 15 & $\begin{array}{l}\text { High-risk } \\
\text { malignancy }\end{array}$ & Imaging grade & Grade 3 & Grade 5 & 0.73 & 0.18 & 3.03 & 0.67 & 0.65 \\
\hline 15 & $\begin{array}{l}\text { High-risk } \\
\text { malignancy }\end{array}$ & Imaging grade & Grade 4 & Grade 5 & 1.22 & 0.25 & 5.91 & 0.80 & 0.65 \\
\hline 16 & $\begin{array}{l}\text { High-risk } \\
\text { malignancy }\end{array}$ & Core biopsy method & Ultrasound & VAB & 1.16 & 0.34 & 3.94 & & 0.81 \\
\hline 17 & $\begin{array}{l}\text { High-risk } \\
\text { malignancy }\end{array}$ & Number of cores & & & 1.05 & 0.89 & 1.24 & & 0.55 \\
\hline 18 & $\begin{array}{l}\text { High-risk } \\
\text { malignancy }\end{array}$ & $\begin{array}{l}\text { Foci of ADH: } \\
\text { continuous }\end{array}$ & & & 0.95 & 0.82 & 1.10 & & 0.49 \\
\hline 19 & $\begin{array}{l}\text { High-risk } \\
\text { malignancy }\end{array}$ & Year of diagnosis & & & 0.91 & 0.77 & 1.08 & & 0.28 \\
\hline 20 & $\begin{array}{l}\text { High-risk } \\
\text { malignancy }\end{array}$ & Patient age & & & 1.03 & 0.97 & 1.09 & & 0.35 \\
\hline 21 & $\begin{array}{l}\text { High-risk } \\
\text { malignancy }\end{array}$ & Episode of screening & & & 1.14 & 1.00 & 1.31 & & 0.05 \\
\hline 22 & $\begin{array}{l}\text { High-risk } \\
\text { malignancy }\end{array}$ & Imaging lesion size & & & 1.01 & 0.99 & 1.03 & & 0.26 \\
\hline
\end{tabular}

Eleven univariate logistic regression models were run to test if any of these covariates were associated with the likelihood of any malignancy.

Subsequently, 11 further univariate logistic regression models were run to test if any of these covariates were associated with the likelihood of high-risk malignancy.

All covariates with $P$-value $<0.2$ on these univariate regression analyses were then retained and evaluated for the multivariable models. 
For predicting the outcome high-risk malignancy, 11 univariate logistic regression models were fitted. The association between the outcome likelihood of high-risk malignancy and episode of screening was not statistically significant at the 0.05 level (global $P$-value $=0.0521$ ), although the $P$-value was close to this threshold. Other covariates tested were not significant.

A multivariable model for the likelihood of highrisk malignancy only contained the covariate screening episode, as it was the only covariate with $P$-value $<0.2$ on univariate regression. A multivariable logistic regression model found this variable was not statistically significant $(P$-value $=0.0521)$.

\section{Discussion}

A diagnosis of atypical ductal hyperplasia based on core biopsy findings of a screen-detected breast lesion represents one of the most common and least controversial indications for diagnostic open biopsy. In our recent audit of 10 years of open biopsy from our large, accredited screening program, atypical ductal hyperplasia was the most frequent indication for open biopsy, representing $14 \%$ of all cases referred for surgery. ${ }^{22}$ In our population-based screening program atypical ductal hyperplasia was diagnosed in 2 of every 1000 women screened and in 1 of 200 women assessed for a screen-detected breast cancer. ${ }^{23}$ The upgrade rate of atypical ductal hyperplasia in our series was $40 \%$, well within the range of $9-56 \%$ reported in contemporary series. Prior studies have identified a range of features as being correlated with the likelihood of malignancy in atypical ductal hyperplasia. These include diagnosis on the basis of $14 \mathrm{G}$ core biopsy, rather than the larger $11 \mathrm{G}$ or $9 \mathrm{G}$ vacuum-assisted core biopsy, ${ }^{6}$ age older than 50 years and mammographic extent $\geq 15 \mathrm{~mm},{ }^{7}$ more than two terminal duct lobular units involved, significant cytological atypia or necrosis, ${ }^{13} \leq 95 \%$ calcifications removed, ${ }^{2}$ three foci of atypical ductal hyperplasia on histology, ${ }^{23}$ extent of atypia, ${ }^{24}$ shorter length of the core biopsy, ${ }^{11}$ the presence of a palpable mass ${ }^{10}$ ipsilateral breast symptoms, suspicious features on sonography or mammography, evaluation by a pathologist with a low volume of atypical ductal hyperplasia diagnoses, concurrent findings of papilloma or radial scar, ${ }^{5}$ or a family or personal history of breast cancer. ${ }^{3}$

Khoury et $a l^{9}$ combined a large number of parameters to present a nomogram for predicting the likelihood of upgrades after a diagnosis of atypical ductal hyperplasia on core biopsy. This nomogram included age, menopausal status, hormone therapy status, personal history of breast cancer, number of involved cores, a solid growth pattern, size of largest focus, and mammographic mass versus calcifications. Although the area under the receiver operator curve was 0.775 , indicating good performance, the predictions of this model are imperfect and the model has not been validated independently. At present, consistent, reliable predictors of malignancy for women diagnosed with atypical ductal hyperplasia on core biopsy remain elusive, such that in many centers a diagnosis of atypical ductal hyperplasia on core biopsy remains an indication for prompt open biopsy, to exclude the presence of concurrent ductal carcinoma in situ or invasive cancer.

Surveillance trials for subsets of ductal carcinoma in situ, currently being launched, will address a knowledge gap regarding the natural history of untreated ductal carcinoma in situ. Particularly in the present context of widespread mammographic screening for breast cancer, the availability of high resolution imaging, the establishment of specific pathologic criteria for the diagnosis and grading of ductal carcinoma in situ, and the demonstration of the efficacy of hormonal therapies in reducing ductal carcinoma in situ recurrence rates after surgery, the need to re-assess the significance of ductal carcinoma in situ in the modern clinical environment is acknowledged. The UK LORIS trial is already underway, whereas the LORD trial, sponsored by the EORTC-Dutch breast cancer group, the COMET trial in the Unites States and the Australian LARRAKIN trial are at various stages of progress.

We can confirm that the preponderance of surgical biopsies performed for a pre-operative diagnosis of atypical ductal hyperplasia, result in non-malignant pathology findings. As such, consideration of inclusion of atypical ductal hyperplasia in trials of active surveillance is valid proposition.

In this series, having evaluated the surgical outcomes for a large cohort of women with screendetected atypical ductal hyperplasia, we have found that adoption of such a strategy would spare four of five women from immediate surgery. According to the LORIS trial design, these women would require annual, rather than 2 yearly imaging follow-up, which is no further impost for this particular cohort, as they are already advised to have annual mammography owing to their increased cancer risk. ${ }^{25}$ However, active surveillance would lead to undertreatment of one in five women who have concurrent but undiagnosed high-risk malignancies, including invasive breast cancer and non-low-grade ductal carcinoma in situ. Although the invasive cancers were sub-centimeter, node negative and ER positive, two of the nine were HER2 amplified, suggesting a potentially aggressive phenotype, for which prompt treatment would usually be advised. In this series, the ductal carcinoma in situ was frequently extensive, leading to multiple surgeries in $29 \%$ of the women, including mastectomy, to achieve clear margins. It is unclear whether observation with annual mammography will adversely affect the survival prospects of the small group of patients with concurrent malignancy associated with atypical ductal hyperplasia. Similar to our findings, Pilewskie et $a l^{26}$ have also reported that $20 \%$ of their 
patients with low-grade ductal carcinoma in situ, eligible for the LORIS trial, had concurrent invasive carcinoma at surgery. The LORIS investigators however reported zero upgrade among the subset of their patients with low-grade ductal carcinoma in situ diagnosed by vacuum-assisted core biopsy. ${ }^{27}$

We, like other researchers, found independent predictors of malignancy among women with atypical ductal hyperplasia diagnosed on core biopsy. Specifically, the review histologic diagnosis and imaging extent of lesion identified lesions more likely to be malignant. The association between the likelihood of malignancy and a more nuanced histological sub-classification of atypia is not unexpected. The histologic definition of atypical ductal hyperplasia is based on findings in surgical specimens. It refers to the involvement of breast ducts by a monotonous proliferation of cells displaying atypia in the range of low-grade ductal carcinoma in situ and showing a cribriform-micropapillary architecture, but falling short of ductal carcinoma in situ due to (1) involvement of less than the entire duct spaces (ie, only partial involvement of the duct spaces), or (2a) involvement of less than two duct spaces (two duct minimum rule) by the characteristic cytologic and architectural changes of cribriform/micropapillary ductal carcinoma in situ, ${ }^{28}$ or (2b) of $<2 \mathrm{~mm}$ in aggregate dimension. ${ }^{29}$ In the more recent context of core biopsy, these quantity criteria cannot be applied reliably. In practice, a broad array of epithelial lesions, displaying varying forms and degrees of atypia, are referred to descriptively as atypical ductal hyperplasia on core biopsy. We agree with UK colleagues that the term atypical intraductal epithelial proliferations is a more appropriate designation for this scenario, as it serves to distinguish them from classic, conventionally defined, atypical ductal hyperplasia. ${ }^{1}$ However, the term atypical ductal hyperplasia is familiar and likely entrenched. Our histologic review confirmed the range of lesions classified descriptively as atypical proliferations on core biopsy. In retrospect, these processes include, at one end of the spectrum, subtle forms of architectural atypia, in the form of micropapillary tufting or minor cribriform proliferations. These foci generally showed minimal cytologic atypia and bordered on florid hyperplasia. At the other end of the spectrum were more extensive alterations, with a complex, cribriform architecture, suggestive of low-grade ductal carcinoma in situ, or exhibiting significant cytologic atypia, exceeding that usually associated with low-grade ductal carcinoma in situ, thus bordering non-low-grade ductal carcinoma in situ. Cases with the cyto-architectural features of classic atypical ductal hyperplasia comprised the largest subset, composing $46 \%$ of the cohort. We found a stepwise increase in the upgrade rate, ranging from $21 \%$, for cases bordering hyperplasia, to $40 \%$ for typical atypical ductal hyperplasia and $69 \%$ when the changes bordered ductal carcinoma in situ due to extent or cytology. The five cases dominated by micropapillary hyperplasia did not include upgrades, but with such few cases, interpretation of this finding is uncertain. Others have found an association between the specific morphology of the atypia and the likelihood of an upgrade. ${ }^{9}$ It is understandable that pathologists would be reluctant to make a definitive diagnosis of ductal carcinoma in situ on core biopsy, opting instead for categorization in the atypical category, since either diagnosis currently results in local excision, with an opportunity to evaluate a larger volume of the breast tissue. The fact that even in cases with cytologic atypia, or with significant architectural complexity that bordered ductal carcinoma in situ, $31 \%$ of the subsequent excisions did not reveal a malignant lesion lends support to this cautious approach.

Although we found statistically significant predictors of malignancy in this series, no reliable predictors could identify cases that were likely to be diagnosed subsequently as having invasive cancer or non-low-grade ductal carcinoma in situ. As such, predicting which patients with a core biopsy diagnosis of atypical ductal hyperplasia may safely opt for yearly surveillance was unreliable. To our knowledge, no other series has addressed this question. Since atypical ductal hyperplasia and low-grade ductal carcinoma in situ represent a morphologic continuum, particularly on core biopsy, these findings likely simulate the disease patterns in women currently being enrolled in ductal carcinoma in situ surveillance trials. Extending the subclassification of ductal carcinoma in situ by molecular techniques, it is possible that molecular genomic analyses for atypical ductal hyperplasia may distinguish subtypes that are precursor lesions to high-grade forms of ductal carcinoma in situ or invasive malignancy.

\section{Conclusions}

Annual surveillance spares $80 \%$ of women unnecessary surgery but presently is not a risk-free option for women diagnosed with atypical ductal hyperplasia on core biopsy, leading to under-treatment of $20 \%$ of this cohort. Although independent predictors of upgrade to malignancy are identified, no features predict high-risk malignancies reliably to assist patient selection for surveillance. In the future, the outcomes from ductal carcinoma in situ surveillance trials are likely to impact the approach to atypical ductal hyperplasia diagnosed by core biopsy.

Open biopsies performed after core biopsy are an important method of last resort for establishing the nature of some breast lesions, which have evaded definitive diagnosis by needle biopsy techniques. Such surgeries find benign processes in most cases. Refinement of the current indications for open biopsy, and non-surgical alternatives for follow-up merit consideration and further research, to reduce the morbidity associated with population-based 
screening. Pooling of large numbers of cases, through collaborative, multicenter studies have the potential to advance knowledge in this field and assist in the appropriate design of trials of surveillance for women diagnosed with atypical ductal hyperplasia on core biopsy.

\section{Acknowledgments}

We acknowledge the contributions of all past and current staff, including medical officers, radiologists, pathologists, and surgeons working at BreastScreen SA and the clients' treating teams outside of the Program. We thank the editorial board and anonymous reviewers of the journal for their assistance in improving the presentation of this work.

\section{Disclosure/conflict of interest}

The authors declare no conflict of interest.

\section{References}

1 Lee Andrew HS, Carder Pauline, Deb Rahul, et al. Guidelines for non-operative diagnostic procedures and reporting in breast cancer screening. The Royal College of Pathologists: 4th Floor, 21 Prescot Street, London, E1 8BB 2016.

2 Allison KH, Eby PR, Kohr J, et al. Atypical ductal hyperplasia on vacuum-assisted breast biopsy: suspicion for ductal carcinoma in situ can stratify patients at high risk for upgrade. Hum Pathol 2011;42:41-50.

3 Ancona A, Capodieci M, Galiano A, et al. Vacuumassisted biopsy diagnosis of atypical ductal hyperplasia and patient management. Radiol Med 2011;116: 276-291.

4 Bendifallah S, Defert S, Chabbert-Buffet $\mathrm{N}$, et al. Scoring to predict the possibility of upgrades to malignancy in atypical ductal hyperplasia diagnosed by an 11-gauge vacuum-assisted biopsy device: an external validation study. Eur J Cancer 2012;48:30-36.

5 Deshaies I, Provencher L, Jacob S, et al. Factors associated with upgrading to malignancy at surgery of atypical ductal hyperplasia diagnosed on core biopsy. Breast 2011;20:50-55.

6 Gumus H, Mills P, Gumus M, et al. Factors that impact the upgrading of atypical ductal hyperplasia. Diagn Interv Radiol 2013;19:91-96.

7 Hong ZJ, Chu CH, Fan HL, et al. Factors predictive of breast cancer in open biopsy in cases with atypical ductal hyperplasia diagnosed by ultrasound-guided core needle biopsy. Eur J Surg Oncol 2011;37: 758-764.

$8 \mathrm{Hsu} \mathrm{HH}$, Yu JC, Hsu GC, et al. Atypical ductal hyperplasia of the breast diagnosed by ultrasonographically guided core needle biopsy. Ultraschall Med 2012;33:447-454.

9 Khoury T, Chen X, Wang D, et al. Nomogram to predict the likelihood of upgrade of atypical ductal hyperplasia diagnosed on a core needle biopsy in mammographically detected lesions. Histopathology 2015;67: 106-120.
10 Kim J, Han W, Go EY, et al. Validation of a scoring system for predicting malignancy in patients diagnosed with atypical ductal hyperplasia using an ultrasoundguided core needle biopsy. J Breast Cancer 2012;15: 407-411.

11 McGhan LJ, Pockaj BA, Wasif N, et al. Atypical ductal hyperplasia on core biopsy: an automatic trigger for excisional biopsy? Ann Surg Oncol 2012;19: 3264-3269.

12 Mesurolle B, Perez JC, Azzumea F, et al. Atypical ductal hyperplasia diagnosed at sonographically guided core needle biopsy: frequency, final surgical outcome, and factors associated with underestimation. AJR Am J Roentgenol 2014;202:1389-1394.

13 Nguyen CV, Albarracin CT, Whitman GJ, et al. Atypical ductal hyperplasia in directional vacuum-assisted biopsy of breast microcalcifications: considerations for surgical excision. Ann Surg Oncol 2011;18: 752-761.

14 Polom K, Murawa D, Kurzawa P, et al. Underestimation of cancer in case of diagnosis of atypical ductal hyperplasia $(\mathrm{ADH})$ by vacuum assisted core needle biopsy. Rep Pract Oncol Radiother 2012;17: 129-133.

15 Francis A, Thomas J, Fallowfield L, et al. Addressing overtreatment of screen detected DCIS; the LORIS trial. Eur J Cancer 2015;51:2296-2303.

16 Elshof LE, Tryfonidis K, Slaets L, et al. Feasibility of a prospective, randomised, open-label, international multicentre, phase III, non-inferiority trial to assess the safety of active surveillance for low risk ductal carcinoma in situ - The LORD study. Eur J Cancer 2015;51:1497-1510.

17 Farshid G, Sullivan T, Downey P, et al. Independent predictors of breast malignancy in screen-detected microcalcifications: biopsy results in 2545 cases. Br J Cancer 2011;105:1669-1675.

18 Thomas J, Evans A, Macartney J, et al. Radiological and pathological size estimations of pure ductal carcinoma in situ of the breast, specimen handling and the influence on the success of breast conservation surgery: a review of 2564 cases from the Sloane Project. Br J Cancer 2010;102:285-293.

19 Farshid G, Kollias J, Grantley Gill P. The clinical utility of assessment of the axilla in women with suspicious screen detected breast lesions in the post Z0011 era. Breast Cancer Res Treat 2015;151:347-355.

20 National Breast Cancer Centre N. Breast Imaging: a guide for practice: 2002 .

21 BreastScreen Australia B. BreastScreen Australia Accreditation Handbook. Commonwealth of Australia: Canberra, 2015.

22 Farshid G, Gill PG. Contemporary indications for diagnostic open biopsy in women assessed for screendetected breast lesions: a ten-year, single institution series of 814 consecutive cases. Breast Cancer Res Treat 2017;162:49-58.

23 Kohr JR, Eby PR, Allison KH, et al. Risk of upgrade of atypical ductal hyperplasia after stereotactic breast biopsy: effects of number of foci and complete removal of calcifications. Radiology 2010;255:723-730.

24 Degnim AC, Dupont WD, Radisky DC, et al. Extent of atypical hyperplasia stratifies breast cancer risk in 2 independent cohorts of women. Cancer 2016;122: 2971-2978. 
25 Degnim AC, Visscher DW, Berman HK, et al. Stratification of breast cancer risk in women with atypia: a Mayo cohort study. J Clin Oncol 2007;25:2671-2677.

26 Pilewskie M, Stempel M, Rosenfeld H, et al. Do LORIS trial eligibility criteria identify a ductal carcinoma in situ patient population at low risk of upgrade to invasive carcinoma? Ann Surg Oncol 2016;23:3487-3493.

27 Soumian S, Verghese ET, Booth M, et al. Concordance between vacuum assisted biopsy and postoperative histology: implications for the proposed Low Risk DCIS Trial (LORIS). Eur J Surg Oncol 2013;39:1337-1340.

28 Page DL, Dupont WD, Rogers LW, et al. Atypical hyperplastic lesions of the female breast. A long-term follow-up study. Cancer 1985;55:2698-2708.

29 Tavassoli FA, Norris HJ. A comparison of the results of long-term follow-up for atypical intraductal hyperplasia and intraductal hyperplasia of the breast. Cancer 1990;65:518-529. 